\title{
Reacciones cutáneas adversas a antimicrobianos sistémicos en pacientes hospitalizados: estudio transversal analítico retrospectivo
}

\author{
Juan Manríquez, Romina Andino-Navarrete, Karina Cataldo-Cerda, \\ Camila Downey-Saldivia y Daniela Berroeta-Mauriziano
}

\section{Inpatient cutaneous adverse reactions to systemic antibiotics: an analytical cross-sectional retrospective study}

Introduction: The reported literature about the types of cutaneous adverse antibiotic reactions (ATB-CAR) and the responsible antimicrobial class is scarce. Aim: to describe the clinical and histopathological profile of these reactions, and potential associations between different types of ATB-CAR and causal antibiotic class in a tertiary hospital in Chile. Material and Methods: Cross-sectional retrospective study performed at the Hospital of the Pontificia Universidad Católica de Chile. Results: A total of 58 patients were included. The most common type of ATB-CAR was morbilliform (n: $37,63.8 \%$ ). The antibiotics most frequently involved were the penicillins and cephalosporins (n: $34,69.3 \%$ ). The most common histological pattern in all types of ATB-CAR was superficial perivascular dermatitis with or without spongiosis. There was significant association between urticarial, morbilliform, DRESS and PEGA types, with the use of penicillins, cephalosporins, cotrimoxazole, and lincomycin, respectively (n: 4,100\%, n: $15,40.5 \%, \mathrm{n}: 2 ; 50 \%, \mathrm{n}: 1,50 \%, \mathrm{p}<0.05$, respectively). Discussion: This is the first description of the ATB-CAR patterns in South American hospitalized patients. Both clinical and histopathological patterns of ATB-CAR are similar to other published series, however the types of causal antibiotics are different.

Key words: South America, cutaneous adverse drug reactions, antibiotics, dermatology.

Palabras clave: América del Sur, reacciones cutáneas adversas a medicamentos, antimicrobianos, dermatología.

\section{Introducción}

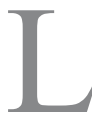

as reacciones adversas cutáneas a medicamentos (cRAM) corresponden al patrón más frecuente de reacción adversa a fármacos, afectando a 2 ó 3\% de los pacientes hospitalizados. Las cRAM secundarias a antimicrobianos (ATM-cRAM) representan un porcentaje alto de estos $\operatorname{casos}^{1,2}$.

Las reacciones adversas a medicamentos generalmente se deben a mecanismos no inmunológicos (idiosincráticos, sobredosis, toxicidad acumulativa, disturbios ecológicos, interacciones con otros medicamentos y activación directa de mastocitos) y, con menor frecuencia, a aquellos que involucran el sistema inmune (incluyen los cuatro tipos de reacciones de hipersensibilidad de Gell y Coombs). Los patrones clínicos de reacción cutánea son variados y no específicos de un determinado medicamento, o mecanismo fisiopatológico subyacente, por lo que es imposible determinar el medicamento causal o el mecanismo involucrado mediante la sola observación clínica.

Los patrones de presentación de las cRAM de tipo inmunológico, varían desde exantemas maculopapulares (EMP), hasta formas graves de vasculitis, pustulosis exantemática generalizada aguda (PEGA), reacción cutánea con eosinofilia y síntomas sistémicos (DRESS), y síndrome de Steven-Johnson/necrólisis epidérmica tóxica (SSJ/NET), asociadas a importante morbilidad y mortalidad $^{1-4}$.

Los estudios epidemiológicos sobre cRAM secundarios a antimicrobianos son escasos, no existiendo reportes de este tipo en población sudamericana ${ }^{2}$.

El objetivo de este estudio fue documentar el perfil clínico, histopatológico, y establecer posibles asociaciones entre los distintos tipos de cRAM y el tipo de antimicrobiano causal, en un hospital universitario en Chile.

\section{Material y Método}

El Departamento de Dermatología del Hospital Clínico de la Pontificia Universidad Católica de Chile (centro docente y de nivel terciario), atiende y mantiene un registro de pacientes hospitalizados, referidos por sus tratantes para evaluación y tratamiento de condiciones cutáneas asociadas, directa o tangencialmente, con el motivo de hospitalización del paciente. Todos los pacientes son evaluados el mismo día de ser referido por el médico tratante.
Pontificia Universidad Católica de Chile. Santiago. Chile. Escuela de Medicina. Departamento de Dermatología.

Financiamiento: No hubo Los autores declaran no tener conflictos de interés.

Recibido: 1 de julio de 2014 Aceptado: 23 de octubre de 2014

Correspondencia a: Juan Manríquez Moreno jjmanriq@gmail.com 
Este estudio fue ejecutado analizando nuestro registro de ATM-cRAM, entre agosto de 2010 y agosto de 2013. En este período, nuestro equipo realizó 1.456 atenciones, representando las ATM-cRAM 4\% del total. La información extraida para este estudio fue obtenida en forma retrospectiva revisando las fichas médicas, fotografías clínicas, análisis de laboratorio e informe de biopsias de piel, de todos los sujetos clasificados como ATM-cRAM en nuestro registro. Dada la naturaleza retrospectiva del estudio, no hubo un criterio pre-establecido para solicitar biopsia de piel, por lo que no en todos los pacientes se dispuso de estudio histopatológico, quedando esta solicitud a criterio del dermatólogo que realizó la primera evaluación. La información de cada sujeto fue extraída y cotejada por dos investigadores, como forma de disminuir la posibilidad de sesgo de información.

Este estudio contó con aprobación del comité de ética institucional.

\section{Causalidad del antimicrobiano}

Para determinar la causalidad del antimicrobiano al cuadro clínico observado se utilizó el algoritmo de Naranjo $^{5}$. Un puntaje en el algoritmo mayor o igual a 5 (equivalente al menos a caso probable) fue utilizado para considerar el cuadro clínico como secundario al uso del medicamento en cuestión. En pocas palabras, el score de Naranjo permite asignar una probabilidad de que el cuadro en observación sea efectivamente secundario a

Tabla 1. Criterios diagnósticos utilizados para clasificar las reacciones adversas a antimicrobianos

\section{Tipo de CRAM* Descripción}

Morbiliforme Máculas diseminadas (lesiones blanqueables a la presión y no palpables, de $1 \mathrm{~cm}$ o menos) y/o pápulas (lesiones palpables y blanqueables de $1 \mathrm{~cm}$ o menos) de aparición eruptiva (días a horas), sin evidencias de compromiso sistémico

DRESS* Criterios del grupo RegiSCAR. Para ser clasificados, los pacientes debieron al menos haber sido catalogados como caso probable (6)

Vasculitis Pápulas purpúricas no blanqueables, sensibles a la palpación, ubicadas en áreas dependientes, con o sin una biopsia de piel compatible, de aparición eruptiva (días a horas)

Urticaria Ronchas y habones pruriginosos diseminados, con menos de $24 \mathrm{~h}$ de duración cada lesión, y no asociados a cambios cutáneos secundarios (hiperpigmentación)

SSJ/NET* Ampollas y erosiones cutáneas diseminadas, comprometiendo entre 1 y $100 \%$ de la superficie corporal, junto a la presencia de compromiso mucoso, presencia de máculas purpúricas y lesiones tipo-target atípicas planas, con mayor acentuación en tronco, en un sujeto seriamente enfermo

RAM fija Máculas o placas eritematosas, redondas, bordes bien definidos, únicas o múltiples, con o sin ampollas, de distribución predominantemente acral. Historia de evento previo en caso recurrente

PEGA*

Criterios del grupo Euroscar. Para ser clasificados, los pacientes debieron al menos haber sido catalogados como caso probable (7)

*Consultar siglas en la Introducción medicamentos (en este caso antimicrobianos), y no a causa infecciosa $u$ otra (enfermedad del tejido conectivo o neoplasias), y que además el medicamento catalogado como responsable sea con alta probabilidad el correcto.

\section{Criterios diagnósticos}

Los criterios diagnósticos utilizados para clasificar los distintos patrones de ATM-cRAM se muestran en la Tabla 1.

\section{Análisis estadístico}

No se calculó tamaño de muestra, pues se analizó el universo completo de sujetos referidos. Se presenta el análisis descriptivo de las variables clínicas, histológicas (en los casos en que se realizó biopsia de piel), y los tipos de antimicrobianos responsables de los distintos tipos de ATM-cRAM. Para determinar posibles asociaciones entre los distintos tipos de cRAM y el tipo de antimicrobiano causal se utilizó test exacto de Fisher. Todas las pruebas fueron a dos colas, considerando significativo un valor p menor a 0,05 .

\section{Resultados}

\section{Tipos de cRAM y tipos de antimicrobianos}

Un total de 58 pacientes con diagnóstico de ATMcRAM fueron incluidos; 26 (48,8\%) eran mujeres. La edad media de presentación fue de $50 \pm 21,4$ años. No se registraron muertes.

El 79,3\% (n: 46) de los casos se inició en forma intrahospitalaria; 77,6\% (n: 45) de los pacientes fueron referidos desde el Servicio de Medicina Interna y Unidad de Cuidados Intensivos, 12,1\% (n: 7) desde el Servicio de Oncología, y sólo 8,6 y 1,7\% desde los servicios de Cirugía y Ginecología-Obstetricia, respectivamente. En 67,2\% (n: 39) de los casos, el diagnóstico de ATM-cRAM fue apoyado por estudio histopatológico.

En relación al tipo de antimicrobiano responsable, las penicilinas (n: $16 ; 38,3 \%$ ), cefalosporinas (n: $18 ; 31,0 \%)$ y carbapenémicos, cotrimoxazol y vancomicina (n: $5 ; 8,6 \%$ cada uno) fueron los más frecuentes.

\section{Asociación entre cRAM y tipo especifico de antimicrobiano}

La cRAM tipo urticaria estuvo significativamente asociada al uso de penicilinas y derivados (p: 0,004; n: 4; 100\%), la cRAM tipo EMP se asoció significativamente al uso de cefalosporinas (p: 0,038; n: 15; 40,5\%; OR 4,1 IC 95\% 1,02 a 16,4). Por otro lado, hubo asociación significativa entre el desarrollo de DRESS y PEGA, con el uso de cotrimoxazol y lincomicina (p: 0,034; n: $2 ; 50 \%$; OR 17 IC 95\% 1,07 a 166,2) y (p: 0,034; n: $1 ; 50 \%)$, respectivamente. 


\begin{tabular}{|c|c|c|c|c|c|c|c|c|}
\hline \multirow[t]{2}{*}{ Tipo } & \multicolumn{2}{|c|}{ Frecuencia } & \multicolumn{2}{|c|}{ Latencia } & \multirow{2}{*}{$\begin{array}{c}\text { Demora } \\
\text { Días (rango)* }\end{array}$} & \multirow{2}{*}{$\begin{array}{c}\text { Medicamentos más frecuentes } \\
\text { Tipo (\%) }\end{array}$} & Biopsia & $\begin{array}{l}\text { Patrones histopatológicos } \\
\text { más frecuentes }\end{array}$ \\
\hline & $\mathbf{n}$ & $(\%)$ & Días & (rango)* & & & n $\quad(\%)$ & Tipo (\%) \\
\hline Morbiliforme & 37 & $(63,8)$ & 7 & $(1-30)$ & $3(0-16)$ & CEF (40,5\%), PEN (24,3\%), GLI (10,8\%) & $24(64,9)$ & DPSCE $(41,7 \%) ;$ INT $(36,1 \%) ;$ EO $(36,1 \%)$ \\
\hline DRESS & 4 & $(6,9)$ & 20,5 & $(10-21)$ & $6,5(0-11)$ & COT (50\%), CEF (25\%), PF (25\%) & $3(75)$ & DPSCE (50\%); VAS (25\%); CAP (25\%) \\
\hline Vasculitis & 4 & $(6,9)$ & 8,5 & $(7-16)$ & $3,5(0-10)$ & PEN (50\%), CEF (25\%), CAR (25\%) & $3(75)$ & $\begin{array}{c}\text { DPSCE (50\%); DPSSE ( } 25 \%) ; \text { INT (75\%); } \\
\text { VAS ( } 25 \%)\end{array}$ \\
\hline Urticaria & 4 & $(6,9)$ & 6 & $(3-30)$ & $0,5(0-2)$ & PEN (100\%) & $3(75)$ & $\begin{array}{c}\text { DPSCE (50\%); DPSSE (50\%); INT (25\%); } \\
\text { EO (50\%) }\end{array}$ \\
\hline SSJ/NET & 3 & $(5,2)$ & 14 & $(6-35)$ & $2(0-4)$ & PEN $(33,3 \%)$, COT $(33,3 \%)$, GLI $(33,3 \%)$ & $3(100)$ & $\begin{array}{c}\text { DPSCE }(33,3 \%) ; \text { DPSSE }(66,7 \%) ; \text { INT } \\
(33,3 \%) ; \text { EO }(66,7 \%)\end{array}$ \\
\hline RAM fija & 4 & $(6,9)$ & 3,5 & $(2-14)$ & $0(0-1)$ & $\begin{array}{c}\text { CEF (25\%), COT (25\%), QUI (25\%), } \\
\text { MAC (25\%) }\end{array}$ & $1(25)$ & DPSSE (25\%); INT (25\%); CAP (50\%) \\
\hline PEGA & 2 & $(3,4)$ & 4,5 & $(1-8)$ & $1,5(1-2)$ & CAR (50\%), LIN (50\%) & $2(100)$ & DPSCE (100\%); EO (50\%) \\
\hline Total & 58 & (100) & 8 & $(0-90)$ & $1(0-60)$ & $\begin{array}{c}\operatorname{CEF}(31 \%), \operatorname{PEN}(27,6 \%), \operatorname{CAR}(8,6 \%), \\
\operatorname{COT}(8,6 \%)\end{array}$ & $39(67,2)$ & DPSCE $(42,1 \%) ;$ INT $(33,3 \%) ;$ EO $(33,3 \%)$ \\
\hline $\begin{array}{l}\text { CEF: cefalosp } \\
\text { de Steven-Jo } \\
\text { INT = derma }\end{array}$ & & $\begin{array}{l}\text { penic } \\
\text { rea } \\
\text { ase, }\end{array}$ & & a a $\mathrm{m}$ & $\begin{array}{l}\text { COT: c } \\
\text { ento. P }\end{array}$ & $\begin{array}{l}\text { I. PF: polifarmacia. DRESS: drug reacti } \\
\text { ulosis exantemática generalizada agu } \\
\text { „litis, CAP=capilaritis. *: Mediana y ra }\end{array}$ & $\begin{array}{l}\text { eosinop } \\
\text { SCE: derr }\end{array}$ & $\begin{array}{l}\text { Ind systemic symptoms. SSJ/NET: síndrome } \\
\text { s perivascular superficial con espongiosis }\end{array}$ \\
\hline
\end{tabular}

Información respecto a la frecuencia de cada ATMcRAM respecto al total, así como la latencia entre el consumo del medicamento y la aparición de lesiones, la demora entre la aparición de las lesiones y la referencia para evaluación, los tipos de antimicrobianos más frecuentemente implicados en cada grupo, así como los patrones histopatológicos más comunes se resumen en la Tabla 2.

\section{Discusión}

Los pacientes con ATM-cRAM atendidos en nuestro hospital son referidos desde el Servicio de Medicina en su mayor parte, y presentan la reacción cutánea como complicación de una hospitalización por otra causa.

La mayoría de los pacientes presentaron EMP, siendo los antimicrobianos causales más frecuentes las penicilinas y cefalosporinas. Las cRAM graves del tipo DRESS, PEGA, vasculitis y SSJ/NET fueron mucho menos frecuentes. Estos hallazgos concuerdan con la mayoría de los estudios publicados, en los que los EMP se reportan como responsables de hasta $90 \%$ de las cRAM, en general ${ }^{8}$.

Con respecto al período de latencia entre el consumo del fármaco y la aparición de lesiones cutáneas, el DRESS fue el grupo que presentó las latencias más prolongadas, hallazgo que es concordante con series publicadas ${ }^{6-8}$.

En relación a los patrones histopatológicos, los hallazgos son concordantes a lo encontrado en estudios previos $^{9,10}$, siendo la dermatitis perivascular superficial, con o sin espongiosis, el patrón más frecuente, seguidos de la presencia de dermatitis con eosinófilos y de dermatitis de interfase. Estos patrones no muestran predilección por algún tipo de ATM-cRAM en particular.

Encontramos asociación estadísticamente significativa entre los patrones urticarial, EMP, DRESS y PEGA, y el uso de penicilinas, cefalosporinas, cotrimoxazol y lincomicina, respectivamente. Nuestros hallazgos son diferentes a los del reciente reporte de Yiang y cols. ${ }^{2}$, realizada en población China, quienes encontraron asociación entre el uso de penicilinas y el desarrollo de SJ/NET, y de glicopéptidos, como causa de DRESS. Estas diferencias podrían ser explicadas por diferentes susceptibilidades genéticas a los distintos tipos de antimicrobianos, y/o por distintos patrones y preferencias en el uso de antimicrobianos sistémicos. No encontramos publicados otros estudios de este tipo para poder comparar nuestros datos.

Las limitaciones de este estudio son inherentes al tipo de diseño. La evaluación de causalidad no fue completa, dada la imposibilidad ética de re-administrar el medicamento sospechoso a los sujetos en estudio y la no realización (por no disponibilidad del recurso) de pruebas inmunológicas in-vivo o in-vitro, para apoyar la asociación causal, las cuales se solicitan según el tipo de cRAM en estudio: en caso de reacciones mediadas por mecanismos de hipersensibilidad tipo I (cRAM de tipo urticariales) se realizan test cutáneos in-vivo o pruebas in-vitro como el RAST (Radio Allergo Sorbent Test), en el que se cuantifican los niveles de IgE sérica específica a los fármacos de interés ${ }^{11,12}$. Por otra parte, en aquellas cRAM cuya fisiopatología depende de una reacción de 
hipersensibilidad tipo IV (PEGA, DRESS, SJ/NET, RAM fija) la causalidad puede ser apoyada in-vivo mediante pruebas de parche positivos y/o pruebas in vitro como el test de activación/transformación linfocitaria ${ }^{13,14}$. Tanto las pruebas in-vivo como in-vitro tienen un rendimiento diagnóstico discreto, y no están disponibles ampliamente.

Adicionalmente, este estudio tiene la limitante fundamental de los estudios retrospectivos que es el posible sesgo en la recolección de datos. Con respecto a los hallazgos histopatológicos, sólo se realizó biopsia a $67,2 \%$ de los pacientes, lo que limita la interpretación de los resultados encontrados.

La aplicabilidad de los datos derivados de este estudio se ve limitada fundamentalmente por la característica intrahospitalaria de nuestro servicio, lo cual puede determinar diferencias en la evaluación de este tipo de pacientes, en otros ambientes hospitalarios que carecen de este recurso.

En conclusión, este corresponde al primer estudio que intenta definir los patrones de ATB-cRAM en sujetos hospitalizados en población sudamericana. Nuestros hallazgos muestran que en nuestro hospital, el tipo más frecuente corresponde al EMP, generalmente secundario a uso de penicilinas y derivados, o cefalosporinas. $\mathrm{Ni}$ los hallazgos clínicos ni histopatológicos son distintos a series publicadas en otras poblaciones; no obstante, las reacciones tipo urticaria, EMP, DRESS y PEGA se ven asociados a tipos de antimicrobianos distintos a los reportados en otras poblaciones.

\section{Resumen}

Introducción: La literatura médica reportada acerca de los tipos de reacciones cutáneas adversas a antimicrobianos (ATM-cRAM) y la clase de antimicrobiano responsable es escasa. Objetivo: Describir el perfil clínico e histopatológico de estas reacciones, y establecer posibles asociaciones entre los distintos tipos de ATM-cRAM y la clase de antimicrobiano causal, en un hospital terciario en Chile. Material y Método: Estudio transversal analítico retrospectivo realizado en el Hospital de la Pontificia Universidad Católica de Chile. Resultados: Fue incluido un total de 58 pacientes. El tipo más frecuente de ATMcRAM fue el morbiliforme (n: 37; 63,8\%). Los antimicrobianos más frecuentemente implicados fueron penicilinas y cefalosporinas (n: 34; 69,3\%). El patrón histopatológico más frecuente en todos los tipos de ATM-cRAM fue el de dermatitis perivascular superficial, con o sin espongiosis. Hubo asociación significativa entre las ATM-cRAM tipo urticaria, morbiliforme, DRESS y PEGA, con el uso de penicilinas, cefalosporinas, cotrimoxazol y lincomicina, respectivamente (n: 4,100\%; n: 15, 40,5\%; n: 2; 50\%; n: 1 ; $50 \%, \mathrm{p}<0,05$, respectivamente). Discusión: Este estudio corresponde a la primera descripción de los patrones de ATM-cRAM en pacientes hospitalizados sudamericanos. Tanto los patrones clínicos como histopatológicos de ATM-cRAM son similares a otras series publicadas; sin embargo, los tipos de antimicrobianos causales no coinciden con lo previamente descrito.

\section{Referencias bibliográficas}

1.- Naldi L, Conforti A, Venegoni M, Troncon M G, Caputi A, Ghiotto E, et al. Cutaneous reactions to drug: An analysis of spontaneous reports in four Italian regions. Br J Clin Pharmacol 1999; 48: 839-46.

2.- $\quad$ Lin Y F, Yang C H, Sindy H, Lin J Y, Rosaline Hui C Y, Tsai Y C, et al. Severe cutaneous adverse reactions related to systemic antibiotics. Clin Infect Dis 2014; 58 (10): 1377-85.

3.- Rahska M P, Marfatia Y S. Clinical study of cutaneous drug eruptions in 200 patients. Indian J Dermatol 2008; 74: 74-80.

4.- Fiszenson-Albala F, Auzerie V, Mahe E, Farinotti R, Durand-Stocco C, Crickx B, et al. A 6-month prospective survey of cutaneous drug reactions in a hospital setting. Br J Dermatol 2003; 149: 1018-22.

5.- Naranjo C A, Busto U, Sellers E M, Sandor P, Ruiz I, Roberts E A, et al. A method for estimating the probability of adverse drug reactions. Clin Pharacol Ther 1981; 30: 239-45.

6.- Kardaun S H, Sekula P, Valeyrie-Allanore L, Liss Y, Chu C Y, Creamer D, et al. RegiSCAR study group. Drug reaction with eosinophilia and systemic symptoms (DRESS): an original multisystem adverse drug reaction. Results from the prospective RegiSCAR study. Br J Dermatol 2013; 169 (5): 1071-80.

7.- Sidoroff A, Dunant A, Viboud C, Halevy S, Bavinck J N, Naldi L, et al. Risk factors for acute generalized exanthematous pustulosis (AGEP)-results of a multinational case-control study (EuroSCAR). Br J Dermatol 2007; 157: 989-96.

8.- Bigby M. Rates of cutaneous reactions to drugs. Arch Dermatol 2001; 137 (6): 765-70.

9.- Mockenhaupt M. Epidemiology of cutaneous adverse drug reactions. Chem Immunol Allergy 2012; 97: 1-17.

10.- Ramdial P K, Naidoo D K. Drug-induced cutaneous pathology. J Clin Pathol 2009; 62 (6): 493-504.
11.- Empedrad R, Darter A L, Earl H S, Gruchalla R S. Nonirritating intradermal skin test concentrations for commonly prescribed antibiotics. J Allergy Clin Immunol 2003; 112 (3): 629.

12.- Fontaine $\mathrm{C}$, Mayorga $\mathrm{C}$, Bousquet $\mathrm{P} \mathrm{J}$, Arnoux B, Torres M J, Blanca M, et al. Relevance of the determination of serum-specific IgE antibodies in the diagnosis of immediate beta-lactam allergy. Allergy 2007; 62 (1): 47.

13.- Barbaud A, Collet E, Milpied B, Assier H, Staumont D, Avenel-Audran M, et al. A multicenter study to determine the value and safety of drug patch tests for the three main classes of severe cutaneous adverse drug reactions. Br J Dermatol 2013; 168 (3): 555-62.

14.- Kano Y, Hirahara K, Mitsuyama Y, Takahashi R, Shiohara T. Utility of the lymphocyte transformation test in the diagnosis of drug sensitivity: dependence on its timing and the type of drug eruption. Allergy 2007; 62 (12): 1439. 